\title{
Information System for Administrating and Distributing Color Images through Internet
}

\author{
Ion LUNGU, Maria ANDRONIE \\ Academy of Economic Studies Bucharest
}

The information system for administrating and distributing color images through the Internet ensures the consistent replication of color images, their storage - in an on-line data base - and predictable distribution, by means of a digitally distributed flow, based on Windows platform and POD (Print On Demand) technology. The consistent replication of color images independently from the parameters of the processing equipment and from the features of the programs composing the technological flow, is ensured by the standard color management system defined by ICC (International Color Consortium), which is integrated by the Windows operation system and by the POD technology. The latter minimize the noticeable differences between the colors captured, displayed or printed by various replication equipments and/or edited by various graphical applications. The system integrated web application ensures the uploading of the color images in an on-line database and their administration and distribution among the users via the Internet. For the preservation of the data expressed by the color images during their transfer along a digitally distributed flow, the software application includes an original tool ensuring the accurate replication of colors on computer displays or when printing them by means of various color printers or presses. For development and use, this application employs a hardware platform based on PC support and a competitive software platform, based on: the Windows operation system, the .NET. development medium and the C\# programming language. This information system is beneficial for creators and users of color images, the success of the printed or on-line (Internet) publications depending on the sizeable, predictable and accurate replication of colors employed for the visual expression of information in every activity fields of the modern society. The herein introduced information system enables all interested persons to access the information from the on-line database, whenever they want, wherever they are, by means of the digital infrastructure, computer networks and modern communication technologies.

Key words: Color management, data bank, web application, Internet, POD, Color space, PCS, CMM, CMS, ICC, CIEXYZ, CIEL*a*b, RGB, CMYK, ICC profile.

\section{D} escription of the Information System In order to be managed and distributed, the data contained by the color publications (complex

color images) need to be processed both at the specific level of the field which they represent and at technical level.

The scientific developments in the fields of color replication and multimedia communications have created the possibility to visually represent information by using color images and enabled the distribution of the color images in electronic format to all interested persons, for viewing them on any available computer's display and/or for printing them at the nearest printer or press.

The modern techniques employed for fast search and identification of the data, which are rapidly being transferred to users from any part of the world, led to the communications globalization through the Internet. The Print On Demand (POD) technology, considered as the technology of the future, is the result of the technological changes in the typographic, editing, multimedia and communications industry, aiming to offer to the clients the color publications they want, where and when they desire. The POD technology allows the integration of certain systems for replication and distribution of color publications, which can be permanently stored in online databases, where they can be viewed directly, by means of the Internet, and replicated on demand. 
As the success of the information expressed by means of color images depends on specifying, replicating and distributing the color perceived by the human visual system, it is needed to integrate certain color management systems that ensure the transfer of color images between the different types of equipment and programs of a replication flow, minimizing, as much as possible, the noticeable differences between the colors captured, displayed or printed by equipments.

The information system for administrating and distributing color images through the Internet is a digital system with color management based on POD (Print On Demand) technology and on the web application administrating the color publications within an on-line data base, which integrates an original mechanism for ensuring color accuracy during their transfer between various hardware and software components of the replication flow.

Structurally, this information system consists of:

- Inputs: color publications (complex color images);

- Processing: creation, up-date and maintenance of the on-line color images data base;

- Outputs: color publications, both displayable or printable by various replication devices (monitors, printers, presses) without distortion or truncation of the information content;

Architecturally, this information system consists of:

- digitally-distributed system for color repli- cation, integrated on Windows platform;

- web application for color images loading in an on-line database and their administration and distribution in electronic format via the Internet.

- color images (color publications) database.

The information system for administrating and distributing color images through the Internet allows creation of on-line color images data bases, more or less complex, which are permanently provided to the users, either for directly viewing them on the computer's display or for printing them on the available printing equipment.

\section{Description of the digitally distributed color replication System}

The information system for administrating and distributing color images through the Internet is implemented through a digitally distributed flow for color replication based on the POD (Print On Demand) technology and on the color management system defined by ICC, which ensures consistent and predictable distribution of color images to various remote users.

The block chart below underlines the main functional blocks of the system and the stages covered by the image files for the achievement of the color transformations required for ensuring their accuracy, when they are transferred between various hardware and software components of the digitally distributed replication flow.

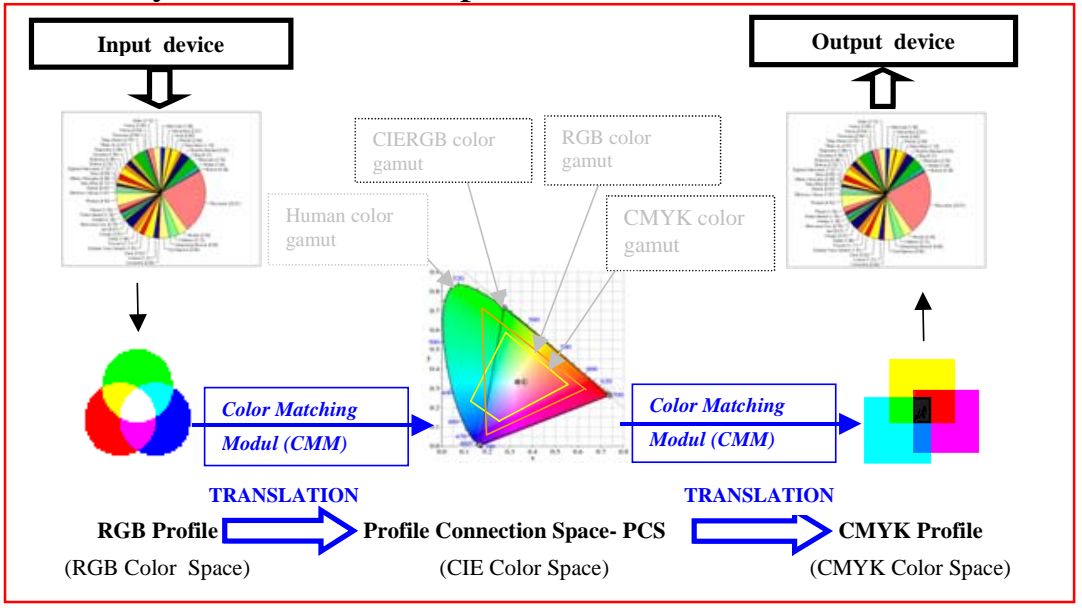


The input equipments (scanner, photo or video digital camera) capture the color images in their own color spaces (the range of the colors that can be replicated, described on basis of a mathematical model, typical for the employed replication technology. The color images thus obtained are transferred in a space of reference color, applying on them the color corrections, specific to each capturing equipment, which define its color profile (the color model or the own features that alter the representation parameters

of the colors from its color range). For this reason, the space of reference color is defined as profile connections space (PCS), where colors from the human scale colors are described in a format which is independent from the parameters of the equipments and programs employed on the technological replication flow.

The color images in PCS format can be stored directly, on magnetic or optical support (hard disk, CD-R/W etc.), for later processing; they can be transferred to various output equipments (monitors, color printers or presses) for viewing or printing; can be undertaken by various graphical applications (Adobe, Corel etc.) for editing and/or compiling with other images/data/texts, or can be distributed via the Internet for distance publication, on web site or in an on-line data base. The software modules for color processing CMM (Color Matching Modules), integrated by the graphical applications and by the operation systems (Windows, Apple etc.) of the computers employed, transfer the color images, from PCS format in the required graphic format and vice-versa, by applying on them the corrections of the color descriptions which define the color profile specific to the hardware and software products employed for their processing.

Translation of the color images from PCS in the color space that depends on the parameters of each output equipment is achieved by applying on them the color profile which defines the model or its features.

The Standard Management System of Colors - CMS (Color Management System) defined by ICC, integrated by the digitally distributed color replication system ensures the consistent and predictable replication of color images when transferring them along the digital technologic flow, consisting of equipments and programs coming from different suppliers. For this, CMS - ICC reports the specific color features of the hardware and software components of the technological flow to its shared reference, with the price of individual transformations for each new component added to the system. It uses as shared reference (PCS) the color spaces defined by the Comission Internationale de l'Eclarage- CIE (CIEXYZ or CIEL*a*b) based on the physical properties of the light and on the features of the software processing applications.

The interlinking system of the color spaces pertaining to the components of the technologic flow for replication by means of a reference space is shown in the chart below.

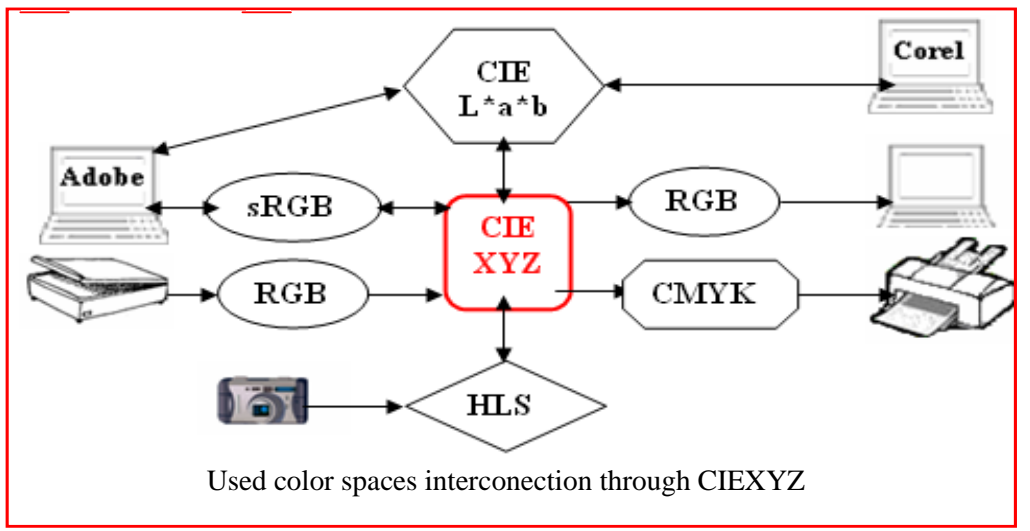

The ICC color profiles make the standard color corrections required for the color images transfer between PCS and the color spaces which depend on equipment or on the graphical application that were imposed as standards in the field (RGB, sRGB, CMYK). For creating ICC profiles pertaining to the equipments and programs of the technologic 
flow, specific programs and calibration devices are employed. CMM- ICC (or ICC compatible) achieves color transformations between source and destination spaces.

For obtaining color images - which can be displayed on monitors or printed on available printers or presses - with maximum efficiency and minimum costs, the system integrates dedicated software modules that stimulate the soft proofing process, according to the production line parameters, based on the ICC profiles and on the specific parameters of each output equipment. These software products enable the user to achieve the necessary color corrections, without viewing or effectively making prints on the destination equipment, so as the image which is viewed on the monitor of a computer to be as much as possible- identical with the one printed by a color printer or press and, within available limits, with the original image.

Developed on basis of the digitally distributed system of color replication introduced, the information system for administrating and distributing color images via the Internet has the advantages offered by using CMS ICC, which ensures the accurate color images replication, by the opportunity to view the images selected from an on-line data base on the monitor of the available computer, if it is connected to the Internet, and by the POD technology, which allows their printing, on demand and at long distance, on own printer or at the nearest printing office.

The information system for administrating and distributing color images through the Internet

ensures the consistent replication of color images, their storage - in an on-line data base
- and predictable distribution, by means of a digitally distributed flow, based on Windows platform and POD (Print On Demand) technology.

\section{Description of the web application for load- ing the color images in an on-line data base, \\ their administration and distribution through the Internet}

The information system for administrating and distributing color images through the Internet integrates a web application with an images data base, designed so as to automatically solve the problems imposed by the consistent replication and predictable distribution of color images, at long distance, in a digitally distributed flow of color production. The functions of this application are designed to enable users' access to the information of the database, ensuring the security, integrity and confidentiality of data.

For development and running, the web application which achieves the administration and distribution color images through the Internet employs a hardware platform based on the PC support, accessible as utilization and costs, and a high performance software platform dedicated to the development of the applications running on the Internet, based on the Windows Operation System (NT/ 2000 server / XP Professional), program language $\mathrm{C \#}$ and development medium .NET., interacting with Microsoft SQL Server 2000 for working with the data base.

The block chart of the web application included in the information system for administrating and distributing color images through the Internet is shown below:

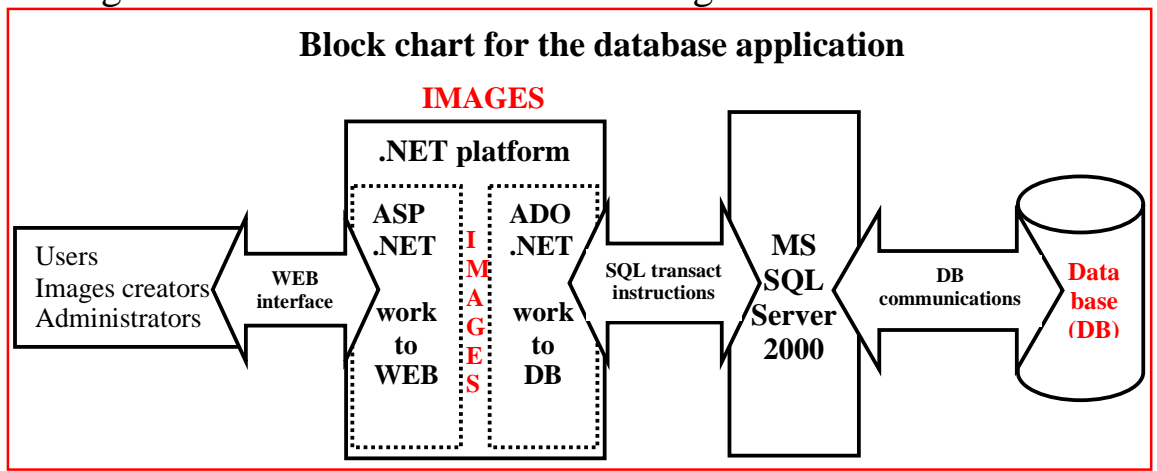

The employment of the ASP.NET technology permitted creating an application with web pages, dynamically generated on a web server, to be transferred to an Internet 
Browser. Therefore, the application installed on a web server can be accessed from any Internet connection by means of the available Internet Browser.

The use of the object categories ADO .NET, offered by the development platform .NET for working with the database, led to creating a software product with disconnected architecture, which eliminates the intensive utilization of the resources when the color images in the database are simultaneously accessed by several users, by copying them on the local machine.

The SQL (Structured Query Language) ensures the handling and finding of data, making possible to take advantage of its facilities in working with the database containing the color images, which are administrated and distributed by the application through the Internet.

The application is used for communication and Internet: Internet Explorer and Internet Information Services, activated for XML applications.

For conserving the information expressed by the color images, when they are transferred along the digitally distributed flow, the software application includes an original mechanism that ensures the accurate replication of the colors when viewed on the computers' monitors, or when printed on various printers or presses. For each color image, the designed software application generates on one side a standard color profile, which it memorizes in the database along with the respective image, and on another side, the color profile that is specific to the employed visualization or printing technologies.

For generating the standard color profile, the standard color transformation defined by ICC is applied on the specific color parameters on the production flow, and for generating the color profiles specific to a replication flow, one must apply the inverse transformations, from the ICC color description to the color descriptions pertaining to the destination equipments.

The following is the Diagram of the database created and administrated by this web application:

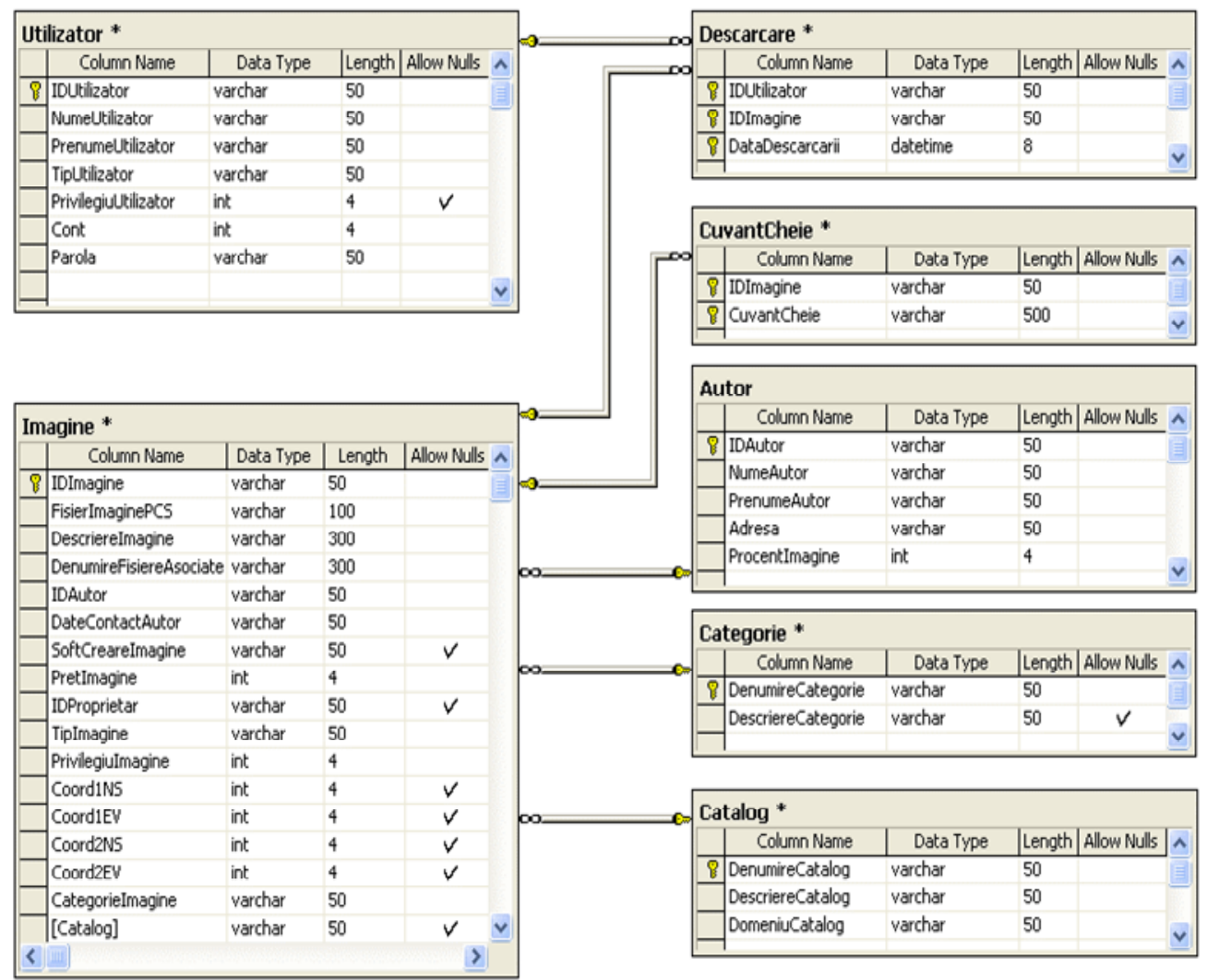

The specifications of the software products employed for its development, the sizeable

quantity of color images stored in the on-line database and the number of users that de- 
mand simultaneous access to the stored images induce the minimum hardware requirements needed by the shown web application, for being able to run.

\section{Functionality of the Information System}

The information system for administrating and distributing color images through the Internet provides the following functions to all the producers, distributors and consumers of data expressed through color images acquired by means of a digital processing technique:

- Taking over he color images from their authors, along with the profiles of the digital system in which they have been created;

- Transformation of the color profiles which accompany the images took over in standard color profiles (ICC);

- Packing and loading of the file sets (images, profiles, execution specifications) in the database;
- Reception of requests for specific images, along with the profile of the digital replication system;

- Generating the specific profiles for the system indicated in the request, starting with the standard from the database;

- Transfer of the requested image to the applicant, along with the pertaining file set, generated for the system to be employed in the replication process;

- Administrating of the images database and providing access with restriction based on user's name and password.

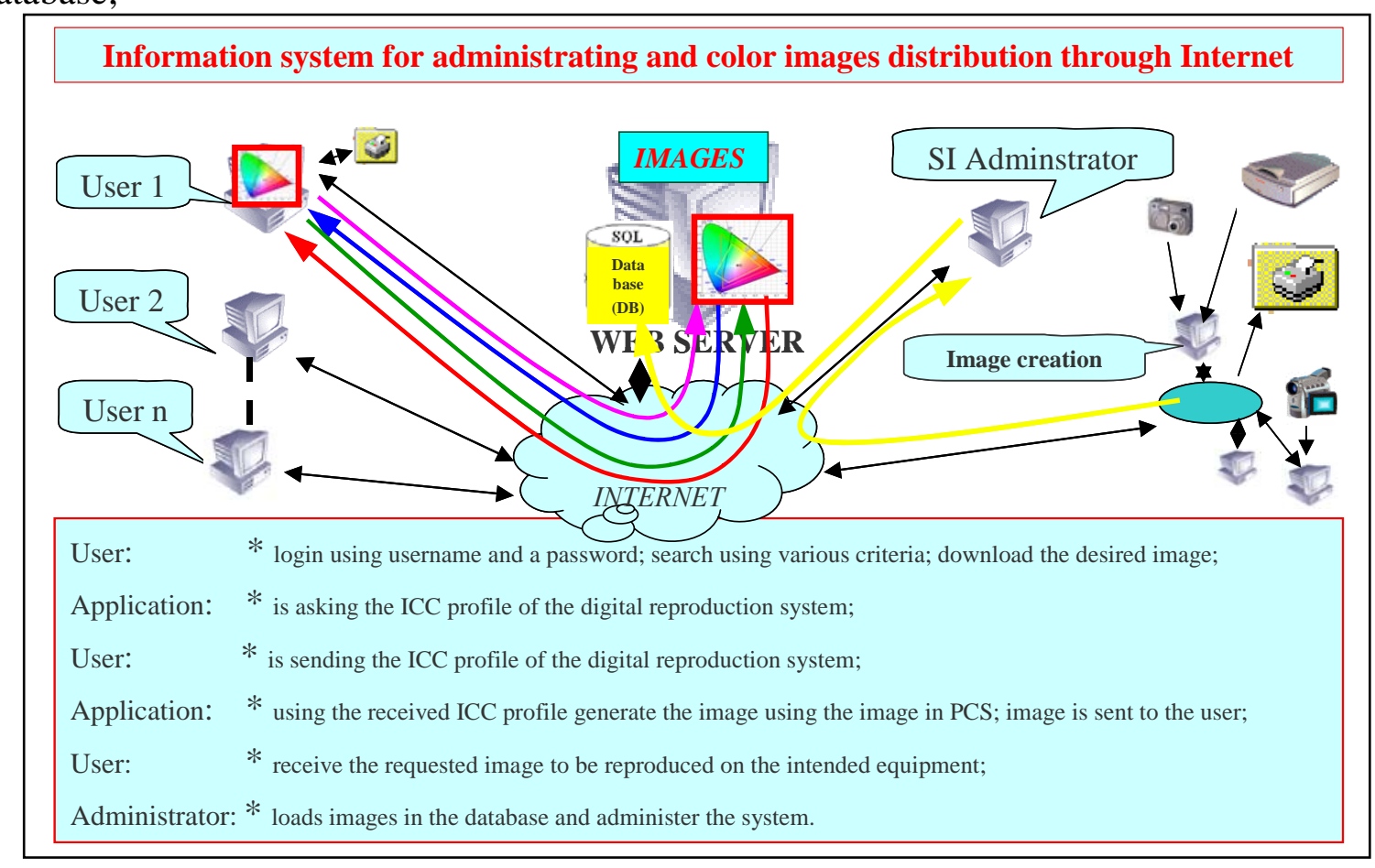

\section{Conclusions}

The information system for administrating and distributing color images through the Internet provides the users a strong tool original thanks to the design solution applied and to its functionality - for working with images and colors.

The solution used for the integration of the information system represents an access way to the data expressed through images and colors which differs from the traditional way, as it is founded on the latest technologies in the fields of color replication, web applications implementation and communications. The originality of this solution resides in the integration modality in a unitary whole of an 
extremely complex information system, on a Windows platform, distributed in a digital flow of POD production with color management based on the standards of technological replication and representation of colors, on which a web application -that achieves administration and distribution of color images processed within the system without color distortions- is run.

The shown information system is flexible, affordable and open. It can be up-dated, both at hardware and software level, by adding high performance equipments, devices and programs for achieving the required aim. Its flexibility and integration ability are due to the utilization of CMS - ICC, considered as the best means of communication of the color between equipments and programs provided by different suppliers, and to the standards for data exchange employed, at industrial level, by the hardware and software producers who use the RGB or sRGB color spaces in the process of capturing and displaying the color images, and the CMYK color space in the process of typing/printing in four colors.

The use of POD technology, accounted -by all those involved in a color replication process - as the most competitive and costeffective integration solution of an information system that generates, administrates and delivers color images, renders to this system all its benefits, enabling it to "deliver" through the Internet color publications to be viewed or printed, even one by one, in the nearest printing workshop in the area or at the user's office.

This information system represents an important documentation source for specialists in various activity fields, ensuring their accesson demand from long distance-to the color publications in their interest areas.

\section{Reference}

1. Phil Green and Lindsay MacDonald, Colour engineering- Achieving device Independent Colour, John Wiley \& Sons SID Series In Display Technology, 2003;

2. Bruce Frase, Fred Bunting, Chris Murphy, Real World Color Management Peachpit Press; 2nd edition (September 8, 2004);

3. *** Microsoft Visual Studio Documentation. 